\title{
Efectos de una sesión de ejercicio aeróbico en la presión arterial de niños, adolescentes y adultos sanos
}

\author{
CRISTIAN ÁLVAREZ ${ }^{1,2, a}$, JAIME OLIVO ${ }^{1}$, \\ OSCAR ROBINSON ${ }^{2, b}$, JOHN QUINTERO ${ }^{2, b}$, \\ VANESSA CARRASCO ${ }^{3, \mathrm{~b}}$, RODRIGO RAMÍREZ-CAMPILLO ${ }^{4, \mathrm{c}}$, \\ DAVID CRISTÓBAL ANDRADE ${ }^{5, \mathrm{~d}}$, CRISTIAN MARTÍNEZ ${ }^{3, \mathrm{e}}$
}

\section{Effects of a single bout of aerobic exercise on body weight and blood pressure among healthy participants}

Background: Physical inactivity is a major risk factor and aerobic exercise is protective for cardiovascular health. Aim: To determine the effects of aerobic exercise on systolic and diastolic blood pressure in children, adolescents and adults. Material and Methods: We studied 13 children aged $10 \pm 1.9$ years, 13 adolescents aged $14.3 \pm 1.5$ and 55 adults aged $41 \pm 11.5$ years. Participants completed 60 minutes of aerobic exercise in one session. Body weight, systolic and diastolic blood pressure, and resting heart rate were measured before and after the exercise period. Results: Adult men and women experienced a significant decrease in body weight after exercise ( -8 and $-6 \%$, respectively). No significant changes in weight were observed in children and adolescents. After exercise systolic and diastolic blood pressure decreased significantly in children (-8.3 and $-5.4 \%$ respectively), adolescents ( -7.6 and $-8.4 \%$ respectively) and adults (-7 and $-5.1 \%$ respectively). Conclusions: $A 60$ minute period of aerobic exercise is associated with a reduction in blood pressure in children, adolescents and adults.

(Rev Med Chile 2013; 141: 1363-1370) tension.

Key-words: Blood pressure; Body weight changes; Exercise; Hyper-

L a hipertensión arterial (HTA) es uno de los principales factores de riesgo que contribuyen al desarrollo de enfermedad cardiovascular, principal causa de muerte en personas adultas $^{1}$ y un importante problema de salud pública ${ }^{2}$. En presencia de sobrepeso, obesidad e HTA, el riesgo de enfermedad cardiovascular es aun mayor, existiendo relación directa entre índice de masa corporal y una mayor tendencia al desarrollo de

\author{
'Centro de Salud Familiar, Los Lagos, Chile. \\ ${ }^{2}$ Facultad de Ciencias de la Actividad \\ Física, Departamento de Educación Física, \\ Universidad San Sebastián, Valdivia, Chile. \\ ${ }^{3}$ Facultad de Educación, Ciencias \\ Sociales y Humanidades, Departamento \\ de Educación Física, Universidad de la \\ Frontera, Temuco, Chile. \\ ${ }^{4}$ Departamento de Ciencias de la Actividad \\ Física, Universidad de Los Lagos, Osorno, \\ Chile. \\ 5Laboratorio de Fisiología Celular, \\ Departamento Biomédico, Facultad de \\ Ciencias de la Salud, Universidad de \\ Antofagasta, Chile. \\ aprofesor de Educación Física, MSc. \\ Entrenamiento Deportivo. \\ bProfesor de Educación Física, MSc. \\ Educación Física. \\ 'Profesor de Educación Física, MSc. \\ Fisiología del Ejercicio. \\ dProfesor de Educación Física, Lic. en \\ Educación Física. \\ eProfesor de Educación Física, MSc. \\ Motricidad Humana. \\ Financiamiento: Este proyecto de \\ investigación fue financiado por fondos \\ del Departamento Ciencias de la Actividad \\ Física de la Universidad de Los Lagos, \\ Osorno, Chile, otorgados a Ramírez R y \\ Álvarez C., y con fondos del Departamento \\ de Salud de la comuna de Los Lagos, Chile. \\ No se declaran conflicto de intereses entre \\ las fuentes de financiamiento en el diseño \\ del presente estudio. \\ Recibido el 16 de octubre de 2012, \\ aceptado el 7 de octubre de 2013 \\ Correspondencia a: \\ Cristian Álvarez Lepín MsC \\ Calle Prat 162, Los Lagos, Región de Los \\ Ríos, Chile. Centro de Salud Familiar de \\ Los Lagos. \\ Teléfono: (56-063) 460314 \\ E-mail: profecristian.alvarez@gmail.com \\ CESFAM Los Lagos, Centro de Promoción \\ de la Salud de la Mujer. \\ Teléfono: (063) 460314
}

$\mathrm{HTA}^{3}$, haciéndose necesario promover el cambio de estilo de vida en la población aún no enferma.

En algunas naciones, la HTA se ha reportado con una prevalencia de 1 a $2 \%{ }^{4}$, aunque en Chile se ha observado que niños y adolescentes han presentado una tendencia a presión arterial elevada de $4,5 \%{ }^{5}$ y cuando se asocia a obesidad como otro factor de riesgo cardiovascular, este valor se eleva a 34\%. La HTA es una enfermedad que no sólo 
afecta la salud y calidad de vida de la población, sino que también esta enfermedad incrementa el costo económico a los sistemas de salud públicos ${ }^{6}$, siendo relevante poder enfatizar en la población sana, los beneficios protectores y terapéuticos de la práctica del ejercicio físico. Se han descrito como principales factores favorecedores de HTA a la inactividad física, dieta (elevada ingesta sodio), consumo de tabaco y factores genéticos ${ }^{7}$, además de un elevado nivel de catecolaminas ${ }^{8}$.

La práctica de ejercicio físico aumenta la presión arterial durante el esfuerzo hasta valores superiores a $200 \mathrm{~mm} / \mathrm{Hg}$ en jóvenes y adultos ${ }^{9}$, pero luego de pocos minutos finalizado el esfuerzo, se produce un efecto hipotensivo tanto en la presión arterial sistólica (PAS) como en la diastólica (PAD). El efecto hipotensivo post ejercicio ha sido reportado en la práctica de ejercicio aeróbico ${ }^{10}$ y de fuerza ${ }^{11}$, en sujetos normotensos y con $\mathrm{HTA}^{12,13}$, pudiendo existir diferencias en cuanto a su magnitud y duración en función del tipo de ejercicio realizado (aeróbico o fuerza) ${ }^{10,11}$, masa muscular y material de ejercicio involucrado (cicloergómetro, treadmill o remoergómetro) ${ }^{14}$, intensidad ${ }^{15} \mathrm{y}$ volumen de ejercicio ${ }^{12,15}$, horario de ejercicio ${ }^{10}$, población y etnia ${ }^{16,17}$ y genética ${ }^{18}$, entre otros (edad, género, fase ciclo menstrual, estado de entrenamiento).

Existen pocos estudios en donde se ha podido estudiar la respuesta de la presión arterial después de una simple sesión de ejercicio físico aeróbico en distintos grupos de edad (niños, adolescentes y adultos), y en su mayoría los reportes han incluído principalmente a sujetos con HTA, de donde se conoce que los efectos del ejercicio físico son mayores respecto a normotensos ${ }^{12}$. Considerando que la práctica de ejercicio físico juega un rol importante tanto en la prevención, mantención y rehabilitación de la salud cardiovascular, el objetivo del presente estudio fue determinar la respuesta de la presión arterial sistólica y diastólica a una simple sesión de ejercicio físico aeróbico en niños, adolescentes y adultos sanos de ambos género y sin HTA.

\section{Material y Métodos}

\section{Participantes}

Mediante invitación pública a participar del estudio se presentaron 129 sujetos, de los cuales sólo 89 cumplieron con los criterios de inclusión. Siete sujetos fueron excluidos del protocolo por inconvenientes con el instrumental de ejercicio (bicicleta), mientras tanto otros cuarenta sujetos fueron excluidos por no corresponder con los criterios de inclusión (padecer HTA, uso de fármacos beta bloqueadores, diabetes mellitus tipo 2). En la muestra final fueron incluidos ochenta y dos sujetos $(\mathrm{n}=82)$ para los análisis estadísticos.

Los criterios de inclusión fueron a) edad entre 7 y 60 años; b) IMC $\leq 35 \mathrm{~kg} / \mathrm{m}^{2}$; c) PAS $<140$ y $\mathrm{PAD}<90 \mathrm{~mm} / \mathrm{Hg}^{19} \mathrm{y}$ d) sin tratamiento con drogas antihipertensivas. Los criterios de exclusión fueron sujetos que declararon a) enfermedad isquémica; b) arritmias, taquicardias; c) enfermedad pulmonar obstructiva crónica y d) ejercicio físico regular ( $\geq 3$ sesiones de $1 \mathrm{~h} /$ semana). Este estudio se realizó considerando la Declaración de Helsinki para estudios con humanos, y fue aprobado por el comité de ética del Centro de Salud Familiar (CESFAM) de Los Lagos, Chile. Todos los participantes dieron su consentimiento por escrito, $y$ en el caso de los menores de edad, lo hicieron sus apoderados y adultos responsables quienes también participaron de la intervención.

El diseño de estudio corresponde al de tipo cuasi experimental y de corte transversal. La muestra incluyó a hijos (alumnos), padres y familiares (apoderados) de cinco establecimientos educativos públicos urbanos (cuatro de nivel escolar primario y uno escolar secundario). Los sujetos fueron asignados intencionalmente a tres grupos, niños (A), adolescentes (B) y adultos (C), siendo distribuidos proporcionalmente hombres y mujeres a cada grupo. Las características de los sujetos se pueden observar en la (Tabla 1).

\section{Protocolo}

Los sujetos se presentaron al lugar de medición una hora antes de intervención, y después de $15 \mathrm{~min}$ fueron evaluados por profesionales enfermeros. Se midió la PAS, PAD y la frecuencia cardiaca en reposo con un esfigmomanómetro digital y automático, previamente validado ${ }^{20}$, marca OMRON $^{\circledR}$ (Modelo HEM-742INT, Bannockburn, Illinois), siguiendo procedimientos estándar ${ }^{19}$. El peso corporal fue medido con una balanza de palanca marca Health o Meter ${ }^{\circledR}$ (Modelo 402 LB, Profesional, USA). Cada sujeto fue medido sin calzado y con ropa deportiva ligera una hora previo ejercicio. Todos los sujetos fueron medidos en un 
Respuesta hipotensiva del ejercicio físico aeróbico - C. Álvarez et al

Tabla 1. Características antropométricas de los sujetos participantes

\begin{tabular}{|c|c|c|c|c|c|c|}
\hline Grupo & $\begin{array}{c}\text { Género } \\
\text { M/H }\end{array}$ & $\begin{array}{c}\text { Edad } \\
\text { (años) }\end{array}$ & $\begin{array}{c}\text { Talla } \\
\text { (m) }\end{array}$ & Test & $\begin{array}{c}\text { Peso } \\
\text { (kg) }\end{array}$ & $\begin{array}{c}\text { IMC } \\
\left(\mathrm{kg} / \mathrm{m}^{2}\right)\end{array}$ \\
\hline \multirow[t]{2}{*}{ Niños (A) } & $M(n=6)$ & $9,5 \pm 1,9$ & $1,42 \pm 0,1$ & $\begin{array}{l}\text { Pre } \\
\text { Post } \\
\Delta \%\end{array}$ & $\begin{array}{c}35,3 \pm 7,4^{B C} \\
35,2 \pm 7,3^{B C} \\
-0,2\end{array}$ & $\begin{array}{c}17,2 \pm 2,6^{B C} \\
17,1 \pm 2,6^{B C} \\
-0,5\end{array}$ \\
\hline & $H(n=7)$ & $10,6 \pm 1,9$ & $1,43 \pm 0,1$ & $\begin{array}{l}\text { Pre } \\
\text { Post } \\
\Delta \%\end{array}$ & $\begin{array}{c}41,4 \pm 10^{B C} \\
41,4 \pm 10,4^{B C} \\
0\end{array}$ & $\begin{array}{c}20,2 \pm 1,7^{\mathrm{BC}} \\
20,3 \pm 1,8^{\mathrm{BC}} \\
+0,4\end{array}$ \\
\hline \multirow[t]{2}{*}{ Adolescentes (B) } & $M(n=6)$ & $14,6 \pm 4,3$ & $1,50 \pm 0,4$ & $\begin{array}{l}\text { Pre } \\
\text { Post } \\
\Delta \%\end{array}$ & $\begin{array}{c}63,4 \pm 5,5^{\mathrm{AC}} \\
63,5 \pm 5,6^{\mathrm{AC}} \\
\quad+0,1\end{array}$ & $\begin{array}{c}24,0 \pm 2,2^{A C} \\
24,0 \pm 2,3^{A C} \\
0\end{array}$ \\
\hline & $H(n=7)$ & $14,1 \pm 1,1$ & $1,58 \pm 0,1$ & $\begin{array}{l}\text { Pre } \\
\text { Post } \\
\triangle \%\end{array}$ & $\begin{array}{c}61,8 \pm 14^{A C} \\
61,6 \pm 14,4^{A C} \\
-0,3\end{array}$ & $\begin{array}{c}24,5 \pm 4,4^{A C} \\
24,5 \pm 4,3^{A C} \\
0\end{array}$ \\
\hline \multirow[t]{2}{*}{ Adultos (C) } & $M(n=25)$ & $41,5 \pm 11,6$ & $1,62 \pm 0,2$ & $\begin{array}{l}\text { Pre } \\
\text { Post } \\
\Delta \%\end{array}$ & $\begin{array}{c}74,9 \pm 8,2^{A B} \\
74,3 \pm 8,3^{A B} \\
-0,8^{*}(P=0,0001)\end{array}$ & $\begin{array}{c}28,5 \pm 4,1^{A B} \\
28,3 \pm 3,7^{A B} \\
-0,7\end{array}$ \\
\hline & $H(n=30)$ & $40,5 \pm 11,5$ & $1,67 \pm 0,4$ & $\begin{array}{l}\text { Pre } \\
\text { Post } \\
\Delta \%\end{array}$ & $\begin{array}{c}77,1 \pm 6,7^{A B} \\
76,6 \pm 6,8^{A B} \\
-0,6^{*}(P=0,0001)\end{array}$ & $\begin{array}{c}27,6 \pm 3,4^{A B} \\
27,4 \pm 3,8^{A B} \\
-0,7\end{array}$ \\
\hline
\end{tabular}

Datos son presentados como media \pm desviación estándar. Grupos de intervención son descritos como: Niños (A), Adolescentes (B) y Adultos (C). Género de los sujetos es descrito como: mujeres (M) y hombres (H). Variable IMC indica: índice de masa corporal. Alndica diferencias significativas $(p<0,05)$ respecto grupo Niños, ${ }^{B}$ respecto grupo adolescentes, Crespecto a grupo Adultos, de acuerdo a ANOVA de una vía. *Indica diferencias significativas $(p<0,05)$ entre pre y post intervención. Las diferencias pre-post fueron analizadas con ANOVA de medidas repetidas.

rango de 60 min antes y después de intervención. Se solicitó a todos los sujetos consumir desayuno habitual previa intervención.

Ejercicio físico aeróbico: Los sujetos recorrieron dos trayectos de $6 \mathrm{~km}$ en bicicleta sobre una superficie plana, a una velocidad $\leq 15 \mathrm{~km} / \mathrm{h}$. Cada trayecto se desarrolló en $30 \mathrm{~min}$, con una pausa de recuperación de 30 min entre ambos recorridos, completando así $60 \mathrm{~min}$ de ejercicio. Cada sujeto utilizó su propio instrumento de ejercicio (bicicleta), los cuales fueron chequeados previo a la actividad por un técnico especialista. Los menores de edad fueron supervisados por sus apoderados o familiares responsables y por educadores físicos quienes acompañaron a los sujetos durante la intervención. Todo sujeto podía desistir de completar la actividad en cualquier momento si lo así lo deseaba, para lo cual adicionalmente se contó con un vehículo especial de transporte, además de personal paramédico en caso de emergencia, cansancio u otra situación que ameritara el cese de la actividad en algún sujeto.
Hidratación: Todos los sujetos fueron provistos con $600 \mathrm{cc}$ de agua mineral con $12 \mathrm{mg}$ de sodio, para ingerir durante y/o después de la intervención, no considerándose esto para efectos de la modificación del peso corporal, pues no fue el objetivo central del estudio.

\section{Análisis estadístico}

Los datos son expresados como media \pm desviación estándar. Todos los datos fueron sometidos a análisis con prueba la de normalidad (Shapiro-Wilk) y de homogeneidad de varianza (Cochran). Para determinar las diferencias iniciales entre grupos se utilizó la prueba ANOVA de una vía, mientras que ANOVA de medidas repetidas (grupos $\mathrm{x}$ tiempo, género $\mathrm{x}$ tiempo $\mathrm{y}$ grupos $\mathrm{x}$ tiempo $\mathrm{x}$ género) fue aplicado, seguido del análisis post hoc de Bonferroni a dos colas para determinar diferencias entre grupos, tiempo y género. Se aplicó la prueba de correlación de Pearson, y eventualmente la prueba de correlación no paramétrica de Tau-b de Kendall. El software 
utilizado fue el paquete estadístico para las ciencias sociales (SPSS) versión 19.0. El nivel alfa para la significancia fue de $\mathrm{p}<0,05$.

\section{Resultados}

Antes y después de la intervención existieron diferencias significativas $(\mathrm{p}<0,05)$ en el peso corporal total e índice de masa corporal entre los grupos niños versus adolescentes y entre niños versus adultos (Tabla 1).

El peso corporal presentó una modificación significativa en el grupo de sujetos adultos de ambos géneros, mientras que el IMC no presentó diferencias significativas después de intervención, (Tabla 1).

Antes y después de la intervención existieron diferencias significativas $(\mathrm{p}<0,05)$ en la PAS y PAD entre los grupos niños versus adolescentes y entre niños versus adultos (Figura 1A).

La PAS disminuyó significativamente en niños $110,1 \pm 3,0$ antes y $100,4 \pm 3,0 \mathrm{~mm} / \mathrm{Hg}$ después $\mathrm{p}=$ 0,0001 , adolescentes $125,3 \pm 3,0$ y $115,4 \pm 3,0 \mathrm{~mm} /$
Hg después $p=0,0001$ y adultos $128,6 \pm 1,4$ y 119,6 $\pm 1,4 \mathrm{~mm} / \mathrm{Hg}$ después $\mathrm{p}=0,0001$. Similarmente la PAD disminuyó significativamente en niños $66,6 \pm 2,1$ antes y $61,0 \pm 2,1 \mathrm{~mm} / \mathrm{Hg}$ después $\mathrm{p}=$ 0,023 , adolescentes $72,3 \pm 2,1$ antes y $66,1 \pm 2,1$ $\mathrm{mm} / \mathrm{Hg}$ después y adultos $74,8 \pm 1,0$ antes y 70,8 $\pm 1,0 \mathrm{~mm} / \mathrm{Hg}$ después (Figura $1 \mathrm{~A}$ ).

El porcentaje de modificación delta de PAS en niños, adolescentes y adultos fue de $-8,6,-6,8$, $-7,9 \%$ respectivamente para cada grupo. Por otra parte, el porcentaje de modificación de PAD en los mismos grupos fue de $-5,4,-8,1$ y $-5,2 \%$, no observándose diferencias significativas entre grupos (Figura 1B).

Considerando a todos los sujetos, cuando éstos se comparan de acuerdo al género, se presentó una menor disminución de la PAS en mujeres $-6,2 \%$ versus hombres $-8,2 \%$, lo cual fue estadísticamente significativo $p=0,037$. En tanto la PAD también disminuyó tanto en mujeres $-5,4 \%$ como en hombres $-6,0 \%$, pero en esta variable no se observaron diferencias significativas entre género (Figura 1B).

Una correlación significativa entre el peso versus la PAS fue observada en niños y adolescentes,

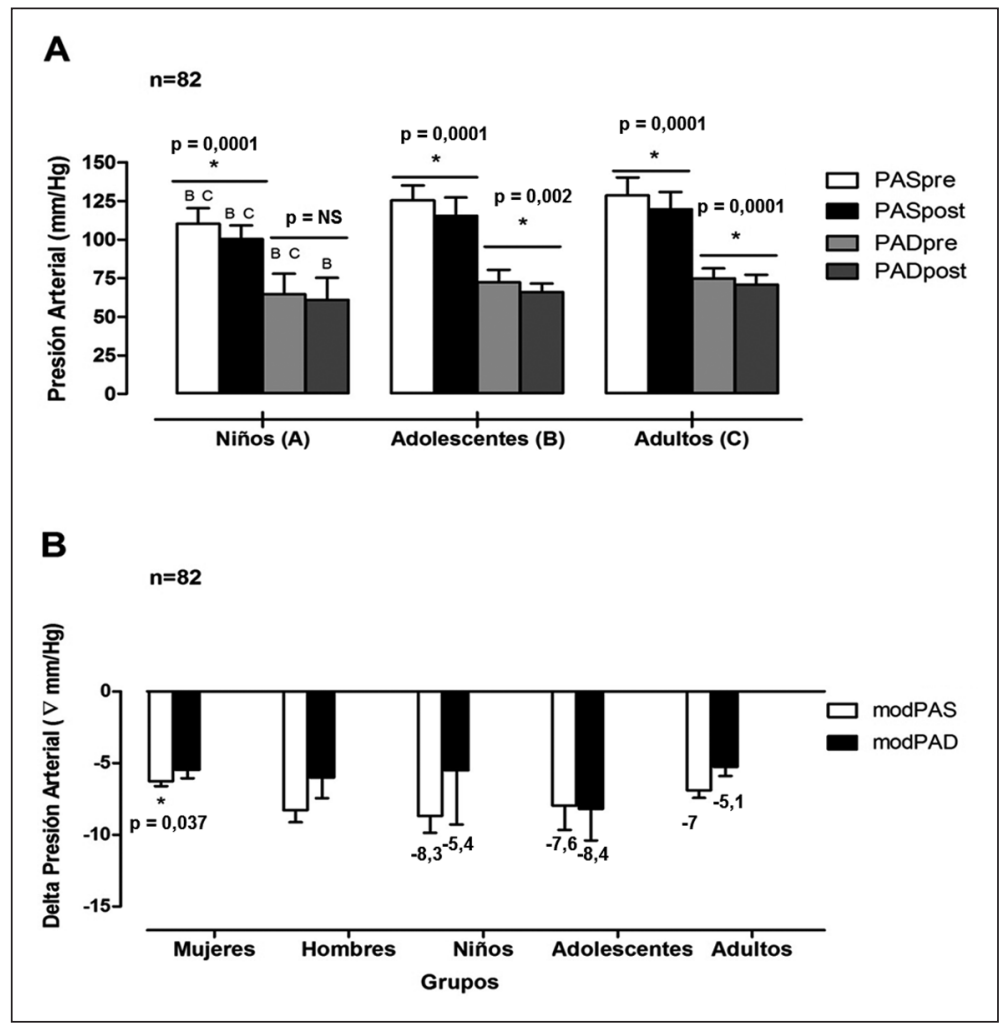

Figura 1. Modificación de la presión arterial sistólica antes (PASpre) y después (PASpost) y presión diastólica antes (PADpre) y después (PADpost), de $60 \mathrm{~min}$ de ejercicio aeróbico en tres grupos de sujetos. Datos son presentados como media \pm error estándar. A. Valores son presentados como $\mathrm{mm} / \mathrm{Hg}$ y los grupos como: Niños (A), Adolescentes (B) y Adultos (C). B. Indica la modificación de la presión arterial sistólica (modPAS) y diastólica (modPAD) en delta porcentual $(\triangle \%)$ entre pre-post intervención. Los grupos son presentados como: Mujeres y Hombres, Niños, Adolescentes y Adultos. Las diferencias previa intervención entre grupos fueron analizadas con ANOVA de 1 vía, mientras que las diferencias en el tiempo con ANOVA de 2 vías. ${ }^{B}$ Indica $(p<0,05)$ respecto a grupo adolescentes, Indica $(p<0,05)$ respecto a grupo Adultos. *Indica diferencias significativas $(p<0,05)$ entre antes y después de intervención. 
Respuesta hipotensiva del ejercicio físico aeróbico - C. Álvarez et al

Tabla 2. Correlaciones bivariadas

\begin{tabular}{|c|c|c|c|}
\hline \multirow{2}{*}{$\begin{array}{l}\text { Pareja } \\
\text { variables }\end{array}$} & \multicolumn{3}{|c|}{ Correlaciones por grupo } \\
\hline & $\begin{array}{c}\text { Niños } \\
r=\end{array}$ & $\begin{array}{c}\text { Adolescentes } \\
\qquad \mathbf{r}=\end{array}$ & $\begin{array}{c}\text { Adultos } \\
r=\end{array}$ \\
\hline Edad-PASpost & $0,45^{*}$ & 0,27 & 0,03 \\
\hline Edad-PADpost & $0,46^{*}$ & $0,61 *$ & 0,01 \\
\hline Pesopre-PASpost & $0,74^{\dagger}$ & $0,63^{*}$ & $0,01 *$ \\
\hline Pesopre-PADpost & $0,72^{\dagger}$ & 0,33 & $0,25^{\dagger}$ \\
\hline PASpre-PASpost & $0,83^{\dagger}$ & $0,78^{\dagger}$ & $0,71^{\dagger}$ \\
\hline PADpre-PADpost & $0,72^{+}$ & 0,38 & $0,63^{\dagger}$ \\
\hline
\end{tabular}

Pesopre: peso corporal antes de ejercicio, PASpre: presión arterial sistólica antes de ejercicio, PADpre: presión arterial diastólica antes de ejercicio, PASpost: presión arterial sistólica después de ejercicio, PADpost: presión arterial diastólica después de ejercicio. ${ }^{*}$ Indica $p<0,05 y{ }^{\dagger} p<0,01$.

así como entre los valores iniciales y después de intervención de las variables PAS y PAD en el grupo adultos (Tabla 2).

\section{Discusión}

El principal hallazgo de este estudio indica que posterior a $60 \mathrm{~min}$ de ejercicio aeróbico de baja intensidad, se presenta un efecto hipotensivo en la presión arterial sistólica (PAS) de niños, adolescentes y adultos. Por su parte la PAD también disminuyó significativamente, aunque sólo en los grupos adolescentes y adultos (Figura 1A).

Encontramos una reducción significativa de PAS $-8,3 \%$ (aprox. $-9,7 \mathrm{~mm} / \mathrm{Hg}$ ) en niños y en adolescentes de $-7,6$ y $-8,8 \%$ para PAS y PAD respectivamente (aprox. $-9,9$ y $-6,2 \mathrm{~mm} / \mathrm{Hg}$ ) ver (Figura 1A). Existen escasos estudios aplicados en niños y adolescentes que han investigado el comportamiento de la presión arterial después de ejercicio físico. En un estudio desarrollado en niños y adolescentes (10 a 17 años) con síndrome metabólico, Chen y cols ${ }^{22}$ reportaron después de intervención con dieta y ejercicio físico una disminución $\sim 10$ y $~ 9 \%$ en PAS y PAD respectivamente. Aunque este estudio duró 2 semanas ( 10 sesiones de ejercicio), sus resultados son similares a las reportadas en el presente estudio, desde el punto de vista del cambio en delta porcentual. Cabe destacar entonces que no considerando el volumen total de sesiones de ejercicio ( 1 sesión o 10 sesiones), la presión arterial presentaría una reducción después de ejercicio de manera independiente del número total (volumen) de sesiones de ejercicio.

Los adultos en tanto, presentaron similarmente una reducción significativa $(\mathrm{p}<0,05)$ en la PAS -7 y PAD - $5,1 \%$ después de intervención, aunque al agrupar a niños, adolescentes y adultos de acuerdo al género, es posible observar una menor reducción de la PAS en mujeres versus hombres, diferencia que fue estadísticamente significativa (Figura 1B).

También en adultos jóvenes sanos, recientemente Chan y cols ${ }^{23}$ reportaron una disminución de PAS y PAD en $\sim 8 \mathrm{~mm} / \mathrm{Hg}$ posterior a $2 \mathrm{~h}$ de haber realizado 20 min ejercicio en bicicleta; MacDonald y cols ${ }^{13}$ observaron después de $70 \mathrm{~min}$ de ejercicio (bicicleta, caminar), la PAS y PAD se reducen en $\sim 16 \mathrm{y} \sim 8 \mathrm{~mm} / \mathrm{Hg}$ respectivamente. Los resultados de ambos estudios previos en adultos son coincidentes con los nuestros, desde el punto de vista de la mayor reducción observada en PAS respecto a la PAD después de ejercicio.

Por otra parte, Williams y cols ${ }^{24}$, después de aplicar $60 \mathrm{~min}$ de ejercicio aeróbico a $60 \%$ del $\mathrm{VO}_{2 \mathrm{MAX}}$ en bicicleta, reportaron la disminución de $-3,4$ y $-2,3 \%$ en PAS y PAD respectivamente, posterior a 45 min finalizado ejercicio. Halliwill y cols ${ }^{25}$, aplicando el mismo ejercicio, reportaron una reducción de $\sim 10 \%$ en el promedio de presión arterial. Los resultados de ambos estudios se dieron paralelo a un incremento de la conductancia vascular y flujo sanguíneo hacia la pierna ejercitada.

Taylor-Tolbert y cols ${ }^{26}$ al estudiar dos grupos de adultos obesos con HTA, reportaron una reducción de 16 y $9 \%$ en PAS y PAD respectivamente (aprox. -7,4 y -3,6 mm/Hg) después de una sesión de 45 min de ejercicio aeróbico (treadmill) a 70\% del $\mathrm{VO}_{2 \mathrm{MAx}}$. Interesantemente estos valores de reducción de la presión arterial se mantuvieron hasta las $24 \mathrm{~h}$ post ejercicio.

Si bien es cierto, sujetos sanos han presentado un efecto hipotensivo post ejercicio en rangos de 5 a $10 \mathrm{~mm} / \mathrm{Hg}^{15,27}$, en pacientes con HTA la evidencia indica que los beneficios del ejercicio físico se incrementan, pues ante un mismo estímulo de ejercicio, sujetos con HTA presentan una mayor magnitud en el efecto hipotensivo en relación a normotensos ${ }^{12,28}$, lo cual tendría implicancia terapéutica a favor del ejercicio. 
Respecto a la duración y magnitud del efecto hipotensor, Jones y $\operatorname{cols}^{10}$ han planteado que este sería más prolongado a mayor duración del ejercicio (30 a $50 \mathrm{~min}$ ), en tanto otros autores han planteado que este se manifestaría independiente de la duración ${ }^{29}$. Llama la atención en un programa regular de ejercicio físico aeróbico de 12 meses (60 min/4 sesiones por semana) aplicado en adultos sedentarios y entrenados, Senitko y cols $^{30}$ reportaron una reducción en rango de $(-4$ a $-5,2 \%$ en PAS) y de $(-3,4$ a $-6,2 \%$ en la PAD), siendo estos resultados similares a los encontrados en el presente estudio posterior a unos $60 \mathrm{~min}$ de ejercicio. A raíz de lo anterior, nosotros planteamos la hipótetis que la variable "cantidad" de ejercicio aplicado no jugaría un rol clave en la magnitud del efecto hipotensivo post ejercicio en sujetos sanos.

Respecto a la intensidad de ejercicio, MacDonald y cols ${ }^{31}$ al probar dos intensidades de ejercicio (50 y $75 \%$ del $\mathrm{VO}_{2 \mathrm{MAX}}$ ) concluyeron que ambas intensidades producen similares reducciones en la presión arterial post ejercicio. De acuerdo con Cornelissen y $\operatorname{col}^{32}$, este efecto tendría explicación en parte por disminución en la resistencia vascular sistémica, aunque otros autores han planteado que esta adaptación podría explicarse por una mejor función endotelial, por la incrementada producción de óxido nítrico y mayor actividad enzimática de la óxido nítrico sintetasa durante ejercicio ${ }^{33,34}$.

También se ha podido observar el rol que juega una mayor temperatura muscular local alcanzada durante la práctica de ejercicio, la cual podría atenuar la respuesta vasoconstrictora cutánea responsable de la disminución del efecto hipotensor cuando se finaliza un esfuerzo ${ }^{35}$, aunque otros autores han descartado esta hipótesis ${ }^{36}$. Otro de los mecanismos potencialmente responsables de la hipotensión post ejercicio es la angiogénesis en el músculo esquelético, definida como la formación de vasos sanguíneos nuevos a partir de otros pre existentes, asociada a una disminución de la resistencia vascular periférica ${ }^{37}$.

La evidencia ha sugerido regularmente, que a un mayor peso corporal e IMC, existirían también niveles más elevados de presión arterial en niños, adolescentes y adultos ${ }^{5,38}$ y por ende estos sujetos presentarían un mayor efecto hipotensivo versus sujetos normopeso. Adicionalmente nosotros encontramos una correlación significativa importante entre el peso corporal inicial de niños versus la PAS y PAD post intervención de este grupo
( $\mathrm{r}=0,74$ y $\mathrm{r}=0,72, \mathrm{P}<0,01)$ respectivamente. También se observó una correlación significativa entre los niveles previos de PAS versus los valores finales de esta misma variable en niños, adolescentes y adultos $(r=0,83, r=0,78$ y $r=0,71$ con $\mathrm{p}<0,01)$ respectivamente, confirmando y coincidiendo con los resultados de estos autores.

Una fortaleza de este estudio fue reportar después de una simple sesión de ejercicio, el comportamiento de la presión arterial en niños, adolescentes y adultos e incluir sujetos de ambos géneros. Dentro de algunas de las limitaciones se encuentra el hecho que la presión arterial sólo se midió en una ocasión, sumado al potencial rol que la dieta (ingesta de café/desayuno) pudo haber jugado en los resultados.

Considerando que la obesidad a nivel escolar en la región de Los Ríos presentó el segundo valor más alto de esta medida respecto la media nacional 23,8\%, y conociéndose que en adolescentes y adultos la situación no es diferente epidemiológicamente, nosotros consideramos necesario enfatizar los beneficios de la práctica de ejercicio físico aeróbico en niños, adolescentes y adultos sanos y como medio de protección de la salud cardiovascular.

Agradecimientos: A Carmen Gloria Flores, Directora del CESFAM de la ciudad de Los Lagos y al personal de apoyo de su establecimiento. A Carmen Fernández, Directora del Hospital Base de la ciudad de Los Lagos y al personal de apoyo de ambulancia. A personal de Carabineros de Chile, quienes participaron de la protección policial de tránsito de los participantes.

\section{Referencias}

1. Pencina MJ, D’Agostino RB, Larson MG, Massaro JM, Vasan RS. Predicting the 30-Year Risk of Cardiovascular Disease. Circulation 2009; 119 (24): 3078-84.

2. Kearney PM, Whelton M, Reynolds K, Whelton PK, He J. Worldwide prevalence of hypertension: a systematic review. J Hypertens 2004; 22 (1): 11-9.

3. Wilsgaard T SHAE. Impact of body weight on blood pressure with a focus on sex differences: The Tromsø study, 1986-1995. Arch Intern Med 2000; 160 (18): 2847 53.

4. Varda N, Gregorič A. A diagnostic approach for the child with hypertension. Pediatr Nephrol 2005; 20 (4): 499506. 
5. Aglony IM, Arnaiz GP, Acevedo BM, Barja YS, Márquez U S, Guzmán A B, et al. Perfil de presión arterial e historia familiar de hipertensión en niños escolares sanos de Santiago de Chile. Rev Med Chile 2009; 137: 39-45.

6. Villarreal-Ríos E, Mathew-Quiroz A, Garza-Elizondo ME, Núñez-Rocha G, Salinas-Martínez AM, GallegosHandal M. Costo de la atención de la hipertensión arterial y su impacto en el presupuesto destinado a la salud en México. Salud Publica Mexico 2002; 44: 7-13.

7. O’Donnell CJ, Lindpaintner K, Larson MG, Rao VS, Ordovas JM, Schaefer EJ, et al. Evidence for Association and Genetic Linkage of the Angiotensin-Converting Enzyme Locus With Hypertension and Blood Pressure in Men but Not Women in the Framingham Heart Study. Circulation 1998; 97 (18): 1766-72.

8. Bertel O, Bühler FR, Kiowski W, Lütold BE. Decreased Beta-adrenoreceptor responsiveness as related to age, blood pressure, and plasma catecholamines in patients with essential hypertension. Hypertension 1980; 2 (2): 130-8.

9. Daida H, Allison TG, Squires RW, Miller TD, Gau GT. Peak Exercise Blood Pressure Stratified by Age and Gender in Apparently Healthy Subjects. Mayo Clin Proc 1996; 71 (5): 445-52.

10. Jones H, Pritchard C, George K, Edwards B, Atkinson G. The acute post-exercise response of blood pressure varies with time of day. Eur J Appl Physiol 2008; 104 (3): 481-9.

11. Polito MD, Simão R, Senna GW, Farinatti PdTV. Hypotensive effects of resistance exercises performed at different intensities and same work volumes. Revista Brasileira de Medicina do Esporte 2003; 9: 74-7.

12. Forjaz CLM, Tinucci Ts, Ortega KC, Santaella DF, Mion DJ, Negrão CE. Factors affecting post-exercise hypotension in normotensive and hypertensive humans. Blood Pressure Monitoring 2000; 5 (5-6): 255-62.

13. MacDonald JR, Hogben CD, Tarnopolsky MA, MacDougall JD. Post exercise hypotension is sustained during subsequent bouts of mild exercise and simulated activities of daily living. J Human Hypertens 2001; 15 (8): 567-71.

14. MacDonald JR MJ, Hogben CD. The effects of exercising muscle mass on post exercise hypotension. J Human Hypertens 2000; 14 (5): 317-20.

15. Jones H, George K, Edwards B, Atkinson G. Is the magnitude of acute post-exercise hypotension mediated by exercise intensity or total work done? Eur J Appl Physiol 2007; 102 (1): 33-40.

16. Pescatello LS, Bairosa L, VanHeest JL, Maresh CM, Rodriguez NR, Moyna NM, et al. Postexercise hypotension differs between white and black women. Am Heart J
2003; 145 (2): 364-70.

17. Sorof JM, Lai D, Turner J, Poffenbarger T, Portman RJ. Overweight, Ethnicity, and the Prevalence of Hypertension in School-Aged Children. Pediatrics 2004; 113 (3): 475-82.

18. Hagberg JM, Ferrell RE, Dengel DR, Wilund KR. Exercise Training-Induced Blood Pressure and Plasma Lipid Improvements in Hypertensives May Be Genotype Dependent. Hypertension 1999; 34 (1): 18-23.

19. Chobanian AV, Bakris GL, Black HR, Cushman WC, Green LA, Izzo JL, et al. Seventh Report of the Joint National Committee on Prevention, Detection, Evaluation, and Treatment of High Blood Pressure. Hypertension 2003; 42 (6): 1206-52.

20. Christofaro DGD, Fernandes RA, Gerage AM, Alves MJ, Polito MD, Oliveira ARd. Validação do monitor de medida de pressão arterial Omron HEM 742 em adolescentes. Arquivos Brasileiros de Cardiologia 2009; 92: 10-5.

21. Donnelly JE, Blair SN, Jakicic JM, Manore MM, Rankin JW, Smith BK, et al. American College of Sports Medicine Position Stand. Appropriate physical activity intervention strategies for weight loss and prevention of weight regain for adults. Med Sci Sports Exerc 2009; 41 (2): 459-71.

22. Chen AK, Roberts CK, Barnard RJ. Effect of a short-term diet and exercise intervention on metabolic syndrome in overweight children. Metabolism: clinical and experimental 2006; 55 (7): 871-8.

23. Chan HH, Burns SF. Oxygen consumption, substrate oxidation, and blood pressure following sprint interval exercise. Appl Physiol Nutr Metab 2013; 38 (2): 182-7.

24. Williams JT, Pricher MP, Halliwill JR. Is postexercise hypotension related to excess postexercise oxygen consumption through changes in leg blood flow? J Appl Physiol 2005; 98 (4): 1463-8.

25. Halliwill JR, Dinenno FA, Dietz NM. $\alpha$-Adrenergic Vascular Responsiveness during Postexercise Hypotension in Humans. The Journal of Physiology 2003; 550 (1): 279-86.

26. Taylor-Tolbert NS, Dengel DR, Brown MD, McCole SD, Pratley RE, Ferrell RE, et al. Ambulatory blood pressure after acute exercise in older men with essential hypertension[ast]. Am J Hypertens 2000; 13 (01): 44-51.

27. Moraes MR, Bacurau RFP, Ramalho JDS, Reis FCG, Casarini DE, Chagas JR, et al. Increase in kinins on postexercise hypotension in normotensive and hypertensive volunteers. Biological Chemistry 2007; p. 533.

28. Bermudes AMLdM, Vassallo DV, Vásquez EC, Lima EG. Monitorização ambulatorial da pressão arterial em indivíduos normotensos submetidos a duas sessões únicas 
de exercícios: resistido e aeróbio. Arquivos Brasileiros de Cardiologia 2004; 82: 57-64.

29. Guidry MA, Blanchard BE, Thompson PD, Maresh CM, Seip RL, Taylor AL, et al. The influence of short and long duration on the blood pressure response to an acute bout of dynamic exercise. Am Heart J 2006; 151 (6): 1322.e5-.e12.

30. Senitko AN, Charkoudian N, Halliwill JR. Influence of endurance exercise training status and gender on postexercise hypotension. J Appl Physiol 2002; 92 (6): 2368-74.

31. MacDonald J, MacDougall J, Hogben C. The effects of exercise intensity on post exercise hypotension. J Hum Hypertens 1999; 13 (8): 527-31.

32. Cornelissen VA, Fagard RH. Effects of Endurance Training on Blood Pressure, Blood Pressure-Regulating Mechanisms, and Cardiovascular Risk Factors. Hypertension 2005; 46 (4): 667-75.

33. Higashi Y, Yoshizumi M. Exercise and endothelial function: Role of endothelium-derived nitric oxide and oxidative stress in healthy subjects and hypertensive patients. Pharmacology \& Therapeutics 2004; 102 (1): 87-96.

34. Higashi Y, Sasaki S, Kurisu S, Yoshimizu A, Sasaki N, Matsuura H, et al. Regular Aerobic Exercise Augments
Endothelium-Dependent Vascular Relaxation in Normotensive As Well As Hypertensive Subjects. Circulation 1999; 100 (11): 1194-202.

35. Low D, Shibasaki M, Davis SL, Keller D, Crandall CG. Does local heating-induced nitric oxide production attenuate vasoconstrictor responsiveness to lower body negative pressure in human skin? J Appl Physiol 2007; 102: 1839-43.

36. Wilkins BW, Minson CT, Halliwill JR. Regional hemodynamics during postexercise hypotension. II. Cutaneous circulation. J Appl Physiol 2004; 97 (6): 2071-6.

37. Ouchi N, Shibata R, Walsh K. AMP-Activated Protein Kinase Signaling Stimulates VEGF Expression and Angiogenesis in Skeletal Muscle. Circ Res 2005; 96 (8): 838-46.

38. Cornelissen VA, Smart NA. Exercise training for blood pressure: a systematic review and meta-analysis. J Am Heart Assoc 2013; 2 (1): e004473.

39. Pescatello L. Exercise and hypertension: Recent advances in exercise prescription. Current Science Inc 2005; 7 (4): 281-6.

40. Haskell W, Lee IM, Pate RR, Powell KE, Blair SN, Franklin BA, Macera CA, Heath GW, Thompson PD, and Bauman A. Physical Activity and Public Health. Circulation 2007; 116 (9): 1081-93. 